\title{
Ironie und Ambiguität: Annäherungen aus literaturwissenschaftlicher Sicht ${ }^{1}$
}

\section{Matthias Bauer}

Ironie und Ambiguität haben offenkundig miteinander zu tun; in welchem Verhältnis sie jedoch zueinander stehen, ist keineswegs geklärt. An einer solchen Klärung soll der vorliegende Beitrag mitwirken. Er beruht auf der Annahme, dass wir aus der Untersuchung der Beziehung von Ironie und Ambiguität etwas über die Natur beider Phänomene lernen können, das uns andernfalls (so) nicht klar wird. Ambiguität hilft m. E. einerseits, zu erklären, worin ironische Äußerungen bestehen bzw. welche Effekte sie haben können, und andererseits hilft die Ironie, bestimmte Dimensionen der Ambiguität besser zu erkennen, die außerhalb lexikalisch/semantisch/syntaktisch beschreibbarer Phänomene liegen, aber trotzdem immer an sprachliche Äußerungen gebunden sind. Es geht also in jedem Fall um die Ironie als Eigenschaft sprachlicher Äußerungen, nicht um so etwas wie „Ironie des Schicksals“ oder „romantische Ironie“, die man als Metaphern des Ironiebegriffs bezeichnen könnte. Deren Verbindung zur Ironie von Äußerungen ist ohne Zweifel ein interessanter Untersuchungsgegenstand, würde aber meinen thematischen Rahmen überschreiten. Ich möchte mich darauf beschränken, anhand einiger literarischer Beispiele verschiedene Formen der Beziehung von Ironie und Ambiguität zu beschreiben. Dabei gehe ich kurz auf den Kontext der Untersuchung ein, das Tübinger Graduiertenkolleg „Ambiguität: Produktion und Rezeption“. Darüber hinaus wird auch die Beziehung des Begriffspaars Ironie und Ambiguität zum Verhältnis von Faktualität und Fiktionalität aufscheinen.

\section{Ambiguität und Ironie als strategische Sprachverwendung}

Dass Ironie und Ambiguität schon seit längerer Zeit als zusammenhängende Phänomene erkannt wurden, kann die Reflexion über einen ironischen Ausdruck belegen, die sich in Daniel Defoes Satire The True-Born Englishman findet. Die Interpretation von Defoes Satire steht hier nicht im Mittelpunkt; aber diese Zeilen, die zugleich eine Reflexion über die Doppelungen enthalten, um die es geht, helfen bei der Bestimmung der Beziehungen von Ironie und Ambiguität.

A True-Born Englishman's a Contradiction,

In Speech an Irony, in Fact a Fiction.

1 Ich danke dem Freiburger Graduiertenkolleg „Faktuales und fiktionales Erzählen“ und insbesondere seiner Sprecherin, Monika Fludernik, für die Gelegenheit, meine Überlegungen zur Diskussion zu stellen. Mein Dank gilt auch dem Tübinger Graduiertenkolleg „Ambiguität: Produktion und Rezeption “ für die Diskussion meiner Überlegungen. Sie sind nicht abgeschlossen. Auch aus diesem Grunde wurde der Vortragsstil weitgehend beibehalten. 
A Banter made to be a Test of Fools,

Which those that use it justly ridicules.

A Metaphor invented to express

A Man a-kin to all the Universe. (Defoe 2003: 95, Z. 372-77)

Der wahre Engländer, wenn ich das so übersetzen darf, ist ein Widerspruch in sich, wobei schon in dieser ersten Zeile Ambiguität ins Spiel kommt, weil nicht klar ist, ob Defoe hier die Redeweise vom „True-Born Englishman“ meint oder die Sache selbst. Der Ausdruck ist in sich ebenso widersprüchlich wie die Sache (impliziert: man kann nicht true-born und ein Englishman sein) ${ }^{2}$ und: der Ausdruck widerspricht der Sache. Wir haben es also mit Ambiguität als Ko-Präsenz mehrerer Bedeutungen zu tun. Beides, Rede und Sache, kommt dann in der zweiten Zeile zur Sprache. True-born Englishman als Ausdruck muss ironisch sein, als Sache inexistent, also eine Fiktion. Damit ist aber auch die Sache selbst eine Rede, und „In Speech an Irony“ kann sich neben dem Ausdruck auch auf die Redeweise des selbstgefälligen Engländers selbst beziehen, die für Defoes satirischen Sprecher ironischerweise gar nicht auf einen true-born Englishman schließen lässt. Wer sich oder andere als „,True-Born Englishman“ bezeichnet, produziert in der Tat eine Fiktion, „in Fact a Fiction“.

In der dritten Zeile wird der Ausdruck, in Übereinstimmung mit der Bezeichnung als Ironie, als „Banter“ bezeichnet, als Neckerei oder Hänselei, die aber nun gerade nicht den Gemeinten verspottet, sondern den, der sie gebraucht: „Which those that use it justly ridicules". Es wird keine genaue Erklärung geliefert, aber mir scheint das zu bedeuten: Wer sich selbst oder jemand anders als „True-Born Englishman" bezeichnet, macht sich dadurch selber lächerlich. Diese Interpretation setzt jedoch voraus, dass der Ausdruck unironisch gebraucht wird. (Diese Lesart bekommen wir, wenn wir „justly“ als nähere Bestimmung von „use“ lesen und nicht von „ridicules“, also use justly als, im exakten Sinn (wörtlich) verwenden ${ }^{3}$ verstehen und nicht justly ridicules als, gerechtfertigterweise lächerlich machen $\left.{ }^{6}\right){ }^{4}$ Eigentlich haben wir dann einen weiteren Widerspruch, denn, unironisch gebraucht, ist „A True-Born Englishman" kein banter (,Scherz') mehr. Aber das kennen wir: Es gibt einen doppelbödigen, ironischen Ausdruck, den der Hörer ernst nimmt und dann in eindeutiger, unironischer Weise als Tatsachenbeschreibung gebraucht. (Beziehungsweise: Eine aufgeblasene Selbstbezeichnung wird ironisch reflektiert, doch der Sprecher merkt das nicht.) Bei Defoe wird solch ein Ausdruck zum Test: Wer ihn ernsthaft gebraucht, macht sich zum Narren. Aber auch: Wird die Ironie als Ironie erkannt oder nicht? Letzteres, die nicht erkannte Ironie, macht den Hörer, wenn er zum Sprecher wird, selbst lächerlich. Was mich hieran interessiert, und zu

2 Im Kontext weist der Sprecher darauf hin, dass „Engländer“ immer schon eine ethnische Mischung waren.

3 OED 1.: „With exactness, exactly, precisely, accurately“; die Bedeutungsmöglichkeit lässt sich durch Intonationswechsel zeigen.

4 Im ersten Fall ist die Segmentierung des Satzes „Which those / that use it justly / ridicules“, im zweiten „Which those that use it / justly ridicules“. 
der Perspektive des Tübinger Graduiertenkollegs führt, ist die Beziehung von Sprecher und Hörer im Hinblick auf Mehrdeutigkeit (vgl. dazu Winter-Froemel/Zirker 2010 und Winter-Froemel/Zirker [im Druck]).

Wir haben im Tübinger Graduiertenkolleg versucht, die Vielfalt der Ambiguitätsphänomene, die in den beteiligten Fächern untersucht werden (neben Linguistik und Literaturwissenschaft verschiedener Philologien sind das auch die Allgemeine Rhetorik, die Neutestamentliche Wissenschaft, die Rechtsgeschichte und die Sozialpsychologie), in einer Matrix abzubilden, die für sich genommen keine neue Theorie zur Ambiguität darstellt, aber hilft, Phänomene der Ambiguität zu beschreiben, zu strukturieren und zu erklären. ${ }^{5}$

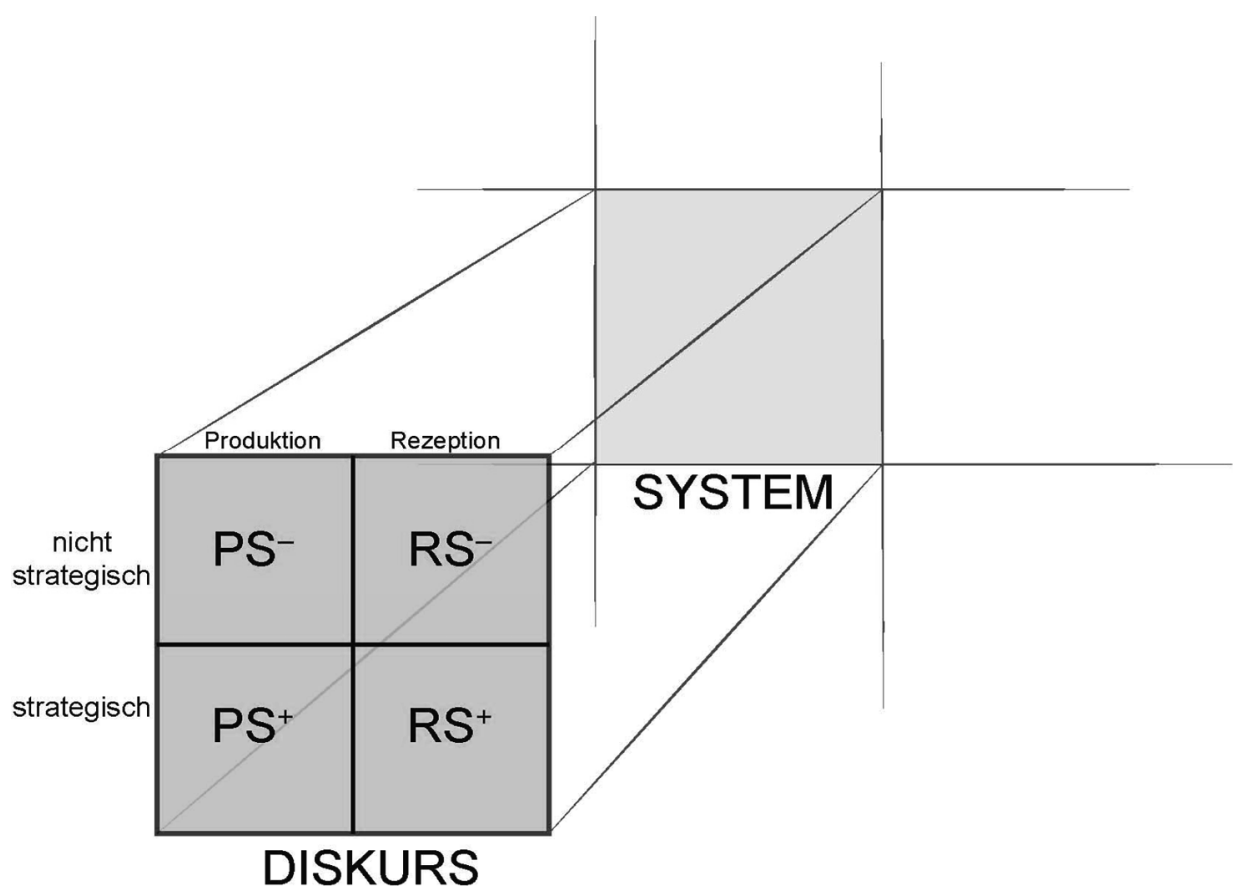

Diagramm: Matrix der Ambiguitätsphänomene $\left(\mathrm{PS}^{+/-}=\right.$Produktion strategisch/nicht strategisch; $\mathrm{RS}^{+/-}=$Rezeption strategisch/nicht strategisch)

Unser Forschungsinteresse speist sich auch aus der Tatsache, dass in linguistischer Hinsicht die strategische Verwendung von Ambiguität bislang eher weniger Aufmerksamkeit erfahren hat, während sie etwa aus rhetorischer und damit auch literaturwissenschaftlicher Perspektive erwartungsgemäß eine zentrale Rolle spielt. So geht es uns darum, Fragen strategischen Gebrauchs in semantische und syn-

5 Siehe auch die weiterführenden Informationen unter www.ambiguitaet.uni-tuebingen.de. Grundlegende Aspekte der Ambiguität, die für das Kolleg eine Rolle spielen, werden beschrieben in Bauer/Knape/Koch/Winkler (2010). 
taktische, sprachhistorische und psycholinguistische Untersuchungen einzubeziehen, und umgekehrt linguistische Analysen und Modelle für die anderen Fächer fruchtbar zu machen. Es erscheint uns dabei produktiv, das Vorhandensein bzw. die Abwesenheit strategischer Sprachverwendung ( $S^{+}$bzw. $\left.S^{-}\right)$im Hinblick auf Mehrdeutigkeit sowohl bei Produzenten (P) als auch bei Rezipienten (R) zu berücksichtigen. So kann es sein, dass Ambiguität überhaupt nur auf Rezipientenseite entsteht, z. B. bei sogenannten mondegreens (Verhörern', akustischen Fehlinterpretationen). ${ }^{6}$ Solche Ambiguitätserzeugung in der Rezeption ist zunächst nicht strategisch, kann es aber werden, etwa wenn ein mondegreen eingesetzt wird, um ein Gespräch in eine bestimmte Richtung zu lenken, und sei es nur zum Zweck der Erheiterung des Gesprächspartners (bzw. der Leser und Zuhörer). Ein Beispiel stammt von P. G. Wodehouse: „,My dear wife ... is taking a trip to the West Indies.' ,Jamaica?" ,No, she went of her own free will““ (Wodehouse 1966: 6). (Wie der Kontext nahelegt, will Lord Ickenham, der auf die Frage „Jamaica?" mit einem Verhörer reagiert, signalisieren, dass es seiner Frau und ihrer Ehe deshalb so gut geht (,[she] continues in the pink“; 6), weil sie ganz freiwillig nach Jamaica gefahren ist.) Hier zeigt sich auch, dass Ambiguität ein Phänomen des Diskurses und kommunikativen Geschehens ist, das von bestimmten systemischen Bedingungen (im vorliegenden Fall etwa der Phonetik oder auch der Gattung der Komödie) abhängt und ggf. auf sie einwirkt.

Bei größeren Texteinheiten können wir ebenfalls von strategischer Ambiguität auf Seiten des Rezipienten sprechen, wenn etwa ein tradierter Text so interpretiert wird, als offeriere er Bedeutungsmöglichkeiten, die der sozialen und ideologischen Realität zur Zeit des Lesers entsprechen. Genauso kann auch die rezipientenseitige Ambiguitätsauflösung oder Vereindeutigung strategischen Gesichtspunkten geschuldet sein, etwa wenn ich einen vieldeutigen Text (einen literarischen Klassiker, einen mehrdeutigen Vertrags- oder Gesetzestext) ausschließlich in dem mir genehmen Sinn auslege.

An diesem Punkt möchte ich noch einmal auf das Defoe-Zitat zurückkommen, weil es in konzentrierter Form einige dieser Aspekte reflektiert. Die in diesem Fall unwissende, unstrategische Ambiguitätsauflösung, das Wörtlichnehmen des Ausdrucks vom „True-Born Englishman“ lässt den Betreffenden lächerlich erscheinen. In unserem Gedichtauszug ist diese Rezipientenhaltung (d. h. die Haltung der Hörer bzw. Sprecher, die den von ihnen wahrgenommenen Ausdruck in einer bestimmten Weise verstehen und entsprechend gebrauchen) nun aber ih-

6 Das $O E D$ erklärt die Entstehung des Ausdrucks folgendermaßen: „the name Lady Mondegreen, a misinterpretation of the phrase laid him on the green in the ballad ,The Bonny Earl of Murray'; coined by S. Wright (see quot. 1954), who introduces the misinterpreted phrase as follows: 1954 S. Wright in Harper's Mag. Nov. 48/1 When I was a child, my mother used to read aloud to me from Percy's Reliques, and one of my favorite poems began, as I remember: Ye Highlands and ye Lowlands, Oh, where hae ye been? They hae slain the Earl Amurray, And Lady Mondegreen." 
rerseits in einer strategischen Ambiguierungsstrategie aufgehoben. Die (mehrdeutige) Redeweise „A True-Born Englishman“ wird vom Sprecher des Gedichtes als Test präsentiert, der diejenigen, die sie nicht durchschauen, als Dummköpfe exponiert (,a Test of Fools"). Die Strategie des satirischen Sprechers besteht also darin, einen gängigen, schlagwortartigen Terminus so zu verwenden, dass seine Bedeutung nicht mehr eindeutig ist, um dann die eindeutige, wörtliche Lesart einem bestimmten Leser- bzw. Sprechertypus zuzuweisen. Wir haben allerdings noch die bereits erwähnte syntaktische Ambiguität der Referenz von ,justly“ zu berücksichtigen, die von der Polysemie dieses Wortes ausgelöst wird: Wenn wir lesen „Which those that use it / justly ridicules“, erhalten wir eine generelle $\mathrm{Zu}$ rückweisung des Ausdrucks true-born Englishman. In dieser Lesart wird die Widersprüchlichkeit („A True-Born Englishman's a Contradiction“) nicht dadurch aufgelöst, dass der Ausdruck ironisch zu verstehen ist. Will sagen: Der Ausdruck bleibt trotz der Ironie widersprüchlich, was syntaktisch durch die einen Kontrast suggerierende Parallele von „In Speech an Irony, in Fact a Fiction“ unterstrichen zu werden scheint. Der Kontrast ist nicht einfach zu konstruieren; ich vermute, er besteht darin, dass die Fiktionalität des Gegenstandes hier als Widerspruch zur ironischen Redeweise aufgefasst werden soll, die ja auf ein Faktum zielt, auch wenn dieses Faktum spöttisch in Frage gestellt werden soll. Wenn ich sage „Du bist ein wahrer Künstler!“, dann gehe ich davon aus, dass es einen solchen gibt; ich sage nur (ironischerweise), dass mein Adressat keiner ist. Bei Defoe wird die Sache selbst, d. h. die Existenz eines genuinen Engländers, als „Fiction“ bezeichnet (d. h. in Abrede gestellt). Deshalb ergibt es keinen Sinn, jemanden ironischerweise als „True-Born Englishman“ zu bezeichnen. Was bleibt, ist die völlige Zurückweisung des Ausdrucks und der Spott über jeden, der ihn gebraucht.

Wir können für den weiteren Zusammenhang festhalten, dass in Ironie Faktualität enthalten ist. Dies geschieht zum einen dergestalt, dass für den Sprecher dasjenige existent sein muss, von dem er ironisch spricht: Es hat keinen Sinn, das Gegenteil des Gesagten als Bezeichnung von etwas Existentem zu meinen (oder auch nur eine signifikante Abweichung vom Gesagten), wenn das Gesagte inexistent ist. ${ }^{7}$ Zum anderen sind ironische Äußerungen nicht fiktional, weil sie sich notwendigerweise auf die Wirklichkeit beziehen. ${ }^{8}$ Sie erfüllen nicht die Bedingung kontrafaktualer Unabhängigkeit, ${ }^{9}$ wie es die Fiktion tut. Im genannten Bei-

7 Wenn in einem Streit jemand zu einem anderen ironisch sagt, „Du bist ein Engel“, so setzt dies voraus, dass es für den Sprecher (und nach seiner Erwartung auch für den Hörer) zumindest das Konzept Engel gibt, damit er den Gesprächspartner so bezeichnen kann.

Vgl. dazu implizit Fludernik (2007: 25). Natürlich sind fiktionale Texte voller ironischer Äußerungen. Gemeint ist, dass ironische Äußerungen nicht diejenige Zugänglichkeitsrelation zur Wirklichkeit haben, die fiktionalen Texten eignet (vgl. dazu Bauer/Beck 2014).

9 Davis spricht unter Berufung auf Currie (1990) von „counterfactual independence requirement“ (2007: 44). Fiktionale Texte können sich z. B. zufällig auf eine real existierende Person oder Lokalität beziehen, ein solcher Wirklichkeitsbezug ist aber nicht notwendig. Diese Unabhängigkeit vom notwendigen Wirklichkeitsbezug ist Merkmal der Fiktion. 
spiel „Du bist ein wahrer Künstler!“ erfinde ich nicht eine Geschichte, in der mein Gesprächspartner ein Künstler ist, sondern ich treffe eine Aussage über meinen Gesprächspartner als Person in der wirklichen Welt. Für Defoe kann ein true-born Englishman in der Tat nur eine Fiktion sein, da der Ausdruck zur Beschreibung real existierender Personen nichts taugt.

Für unsere Reflexion ist nicht unwichtig, dass der mehrdeutige Ausdruck bzw. die Sache, die er bezeichnet, nicht nur als „Contradiction“, „Irony“ und „Fiction“ bezeichnet wird, sondern schließlich auch als „Metaphor“. Angeblich soll nun der Ausdruck true-born Englishman metaphorisch einen Menschen bezeichnen, der dem Universum verwandt ist. Der Kontext lässt wenig Zweifel an der Ironie dieser Aussage. Wieder spielt Ambiguität mit, die nun den wahren Sinn des ironisch als „man a-kin“ Bezeichneten enthüllt: Hier wird ein Verhörer nach Art des Mondegreen strategisch provoziert, so dass ein „manakin“ (engl. manikin; deutsch Männeken) herauskommt: mehr ist der true-born Englishman nicht.

Diese Verbindung von Ironie und Ambiguität in den letzten beiden Zeilen unseres Defoe-Zitats führt direkt zu unserer Hauptfrage nach dem Verhältnis von Ironie und Ambiguität, das meines Erachtens, trotz einer Fülle von Arbeiten zu Ironie und zu Ambiguität, nicht wirklich geklärt ist. Meine Diskussion hat bislang offenbar stillschweigend vorausgesetzt, dass Ironie mehrdeutig ist, aber ob und wie das der Fall sein kann, ist keineswegs ausgemacht. So gibt es in verschiedenen Fach- oder Diskurszusammenhängen die durchaus nachvollziehbare Auffassung, dass Ironie deutlich von Ambiguität zu scheiden sei. Zurückgehend auf die klassische Rhetorik wird Ironie häufig so definiert, ${ }^{10}$ dass ein ironischer Ausdruck das Gegenteil dessen bedeutet, was er besagt; dies findet sich etwa bei Quintilian als Definition der Ironie als Form der Allegorie „in quo contraria ostenduntur" (8.6.54) (daher auch die Verbindung zur Metapher). Damit ist der ironische Ausdruck aber alles andere als mehrdeutig; er kann sogar kommunikativ auf Klarstellung bzw. Vereindeutigung abzielen (vgl. Lausberg 1973: Par. 582). ${ }^{11}$ In ganz anderer Perspektive, aber mit ähnlichem Resultat wendet sich Linda Hutcheon (1994) gegen die Verbindung von Ironie und Ambiguität, wenn sie betont: „But ambiguity and irony are not the same thing: irony has an edge" (33). Sie ist vor allem an Ironie als einem politischen Instrument interessiert, das spezifische Effekte auslöst, und muss deshalb der Ambiguität reserviert gegenüberstehen bzw. ihr klare Grenzen setzen: „My interest here [...] is in irony, not ambiguity“ (70). Schon

10 Zur Geschichte der Definitionen von Ironie vgl. den Überblick bei Behler (1998).

11 In ähnlicher Weise betont Booth (1974) die Eindeutigkeit ironischer Aussagen: „But the irony that disorients by resisting univocal interpretation, the irony that evades committed speech, is only a branch of a great and ancient art; even those modern works which are rightly celebrated for their rich ambiguity reveal, on close inspection, large tracts of stable irony [...] about which no careful reader experiences any ambiguity whatsoever" (48; vgl. 127). 
Quintilian fügt an der zitierten Stelle hinzu, dass Ironie eine Form der inlusio oder illusio ist, also der Verspottung (8.6.54); dem entspricht Hutcheons „edge“.12

Allerdings können wir m. E. dem Phänomen der Ironie ohne eine systematische Einbeziehung der Ambiguität nicht gerecht werden, und umgekehrt ist Ambiguität, wie ich an der Matrix zu zeigen versucht habe, ein Äußerungsphänomen, das vielfach strategisch eingesetzt und interpretiert wird. Was von Hutcheon als „edge“ bezeichnet wird, ist ein Beispiel für eine solche Strategie, auch wenn es natürlich noch ganz andere Strategien des Einsatzes von Ambiguität gibt, etwa die erwähnte Etablierung einer bestimmten Bedeutung in einem kanonischen oder normativen Text, oder auch die gezielte Verunsicherung. Hutcheon selbst benötigt die Ambiguität für ihre Ironiekonzeption. ${ }^{13}$ Dies wird deutlich, wenn sie sagt: „Ambiguity does not depend on the simultaneous and edgy playing off of one meaning against another in a relational, inclusive, and differential way [...]; irony does" (70; Hervorhebung im Original). Hier findet eine kategoriale Vermischung statt; frech und kantig zu sein, gehört zur Ironie, der Gebrauch verschiedener Bedeutungen gehört zur Verwendung von Ambiguität. Wenn ich verschiedene Bedeutungen derselben Äußerung (oder derselben Sache) gegeneinander ausspiele, arbeite ich mit Ambiguität in einer spezifischen Weise. So deutet es auf eine strategische Ambiguitätsverwendung hin (in der obigen Matrix $\mathrm{PS}^{+}$), wenn Bedeutungen gegeneinander ausgespielt werden. Die zitierte Beschreibung der Ironie setzt also sogar Ambiguität voraus, definiert sie (ohne dass dies explizit gesagt würde) als eine spezielle Form der Ambiguitätsverwendung. Ich würde gar nicht so weit gehen. Wenn jemand mitten in einem Streit sagt, „wunderbar, wie gut wir uns wieder verstehen“, so ist das ironisch (und „edgy“) aber nicht mehrdeutig; es werden nicht wirklich verschiedene Bedeutungen gegeneinander ausgespielt. Übrigens fällt hier die Negativität der Kontrastbedeutung auf, wie sie ja auch beim „True-Born Englishman“ zu sehen ist; es ist sehr viel schwerer, wenn auch nicht unmöglich, eine positive ironische Bedeutung zu etablieren („schade, dass wir uns wieder so schlecht verstehen“, wenn gerade ein Moment völligen Einverständnisses herrscht). Nicht jede Mehrdeutigkeit ist ironisch und nicht jede ironische Sprachverwendung bringt Ambiguität mit sich.

12 Vgl. dazu auch Sperber/Wilson: „The attitude expressed by an ironical utterance is invariably of the rejecting or disapproving kind“ (1995: 139). Allerdings geht diese Aussage sehr weit; ironische Äußerungen können auch z. B. freundschaftlichen Spott bedeuten.

13 So widmet auch Booth einen beträchtlichen Teil seines Buches den mit Ironie verbundenen „[i]nstabilities“ (Booth 1974: 230-77). Er unterscheidet dabei verschiedene Grade der Offensichtlichkeit von Ironie sowie Grade der Stabilität erkannter Ironie (ist der Leser „fertig“, wenn er die Ironie erkannt hat?). Mit der hier erkennbaren Verbindung zur Ambiguität hat Booths dritte Kategorie der „[i]nstability“ allerdings systematisch nichts zu tun, nämlich der Umfang dessen, was ironisch unterminiert wird (von lokaler Ironie bis hin zu „absolute infinite negativity“) (234). 


\section{Ambiguität und Ironie: Drei Arten der Beziebung}

Wie verhält sich dann aber Ironie zu Ambiguität? Es dürfte klar sein, dass die beiden Phänomene zwar nicht miteinander identisch, aber aufeinander bezogen sind und dass es für das Verständnis kommunikativer Prozesse (und ich beschränke mich hier auf die Sprache) wichtig ist, das Verhältnis zu klären. Ich kann das nicht umfassend und abschließend tun, möchte aber doch wenigstens ansatzweise zu einer Systematisierung beitragen, indem ich drei Beziehungsdimensionen zur Diskussion stelle.

1. Ambiguität aufgrund ironischer/nicht ironischer Bedeutung einer Äußerung 1.1 Es ist nicht eindeutig zu bestimmen, ob eine Äußerung ironisch ist oder nicht, entweder weil die Ironiesignale nicht ausreichend oder eindeutig sind oder weil es trotz dieser Marker zur ironischen Lesart starke Alternativen gibt. Es handelt sich nicht um Vagheit, weil sowohl die ironische als auch die nicht-ironische Bedeutung voneinander geschieden sind und im Kontext Sinn ergeben (s. u. das Polonius-Beispiel).

1.2 Ironische und nicht ironische Bedeutung sind gleichzeitig präsent oder zeitlich distant innerhalb desselben Äußerungszusammenhanges (z. B. eines literarischen Textes), sie sind aber auf verschiedene Kommunikationspartner bzw. -ebenen verteilt (z. B. Figur und Zuschauer bei dramatischer Ironie) (s. u. Great Expectations-Beispiel); es kann sich auf der einen Ebene um eine fiktionale, auf der anderen um eine nichtfiktionale Äußerung handeln.

2. Ambiguität aufgrund der zusätzlichen kommunikativen Bedeutung einer ironischen Äußerung

Es kann offen bleiben, ob primär die propositionale Bedeutung der ironischen Äußerung gemeint ist (also häufig oder meistens das Gegenteil des Gesagten) oder ob die Bedeutung der ironischen Äußerung als illusio/Verspottung („edge") im Vordergrund steht.

3. Ambiguität aufgrund des Simulationscharakters einer ironischen Äußerung Die Vortäuschung einer Aussage/Meinung/Einstellung geht einher mit der Sichtbarmachung der Vortäuschung.

\section{Ambiguität aufgrund ironischer/nicht ironischer Bedeutung einer Äußerung}

Als erstes sehe ich Ambiguität im Spiel in jener besonderen Form, die unterschiedliche Kommunikationspartner bzw. Kommunikationsebenen betrifft und/ oder die als ein dynamisches, von zeitlicher Entwicklung abhängiges Phänomen erscheint. Um noch einmal auf unser Beispiel zurückzukommen: Es gibt einen Ausdruck, true-born Englishman, der vom Sprecher ironisch gemeint ist, der aber nicht von allen so verwendet wird („those that use it justly"). Die ironische Bedeutung ist nicht lexikalisiert (etwa im Unterschied $\mathrm{zu}$ manchen figurativen 
Wortbedeutungen), ${ }^{14}$ trotzdem können im Kommunikationsprozess beide nebeneinander existieren. Die Natur des ironischen Sprachgebrauchs - es wird nicht das gemeint, was gesagt wird; das Gemeinte muss übrigens nicht unbedingt das genaue Gegenteil des Gesagten sein, damit wir von Ironie sprechen - bringt es mit sich, dass ein Kommunikationspartner u. U. das Gesagte als das Gemeinte versteht, so dass im Endeffekt zwei Bedeutungen im Kommunikationsprozess gleichzeitig präsent sind, die ironische des Sprechers und die wörtliche des Hörers. Im Falle Defoes sehen wir als Leser beide Bedeutungen bzw. der Sprecher des Gedichts führt sie uns in einer Art Metadiskurs vor Augen.

Aber das ist nicht immer der Fall, was insbesondere bei literarischen Texten dazu führt, dass es unterschiedliche Interpretationen gibt. So kann z. B. hörerseitig Ironie wahrgenommen werden, während diese sprecherseitig womöglich gar nicht vorliegt. Ein Beispiel dafür ist folgender Auszug aus dem Dialog von Ophelia und ihrem Vater Polonius, in dem dieser seine Tochter über ihre Beziehung zu Hamlet ausfragt: ${ }^{15}$

OPHELIA He hath, my lord, of late made many tenders

Of his affection to me.

POLONIUS Affection? Pooh, you speak like a green girl,

Unsifted in such perilous circumstance.

Do you believe his tenders, as you call them?

OPHELIA I do not know, my lord, what I should think.

POLONIUS Marry, I will teach you. Think yourself a baby

That you have ta'en these tenders for true pay

Which are not sterling. Tender yourself more dearly

Or-not to crack the wind of the poor phrase,

Running it thus-you'll tender me a fool. (1.3.99-109)

Während Ophelia das Wort „tender“ hier im Sinne einer Liebesgabe gebraucht (die Zeile wird angeführt unter $O E D, 2$. gen. An offer of anything for an acceptance"), und bei ihr deutlich der Sinn des Adjektivs tender ,zart' mitschwingt, verwendet Polonius, besonders in Zeile 106, das Wort im kommerziellen Sinn $(O E D$ „1. spec. An offer of money, or the like, in discharge of a debt or liability"). Es ist aber keineswegs klar, ob Polonius hier ironisch spricht, d. h. das Wort Ophelias aufgreift und in einem Sinn verwendet, der das Liebesverhältnis in sein Gegenteil, ein kommerzielles Verhältnis verwandelt; in diesem Fall würde es sich

14 Ein Beispiel ist true-born: Wenn der Ausdruck im ironischen Sprachgebrauch eine negative Bedeutung bekommt, so findet sich diese nicht im Lexikon. Demgegenüber ist z. B. die figurative Bedeutung „having the sterling qualities associated with such [i.e. pure] descent“ im $O E D$ zu finden. Diese ist aber nicht die ironische Bedeutung. Davon unabhängig ist es aber natürlich möglich, figurative Wortbedeutungen ironisch zu verwenden (s. u. das Polonius-Beispiel).

15 Diese Passage wird (u. a. im Hinblick auf Ambiguität und die Figur des Polonius in verschiedenen Fassungen des Textes) detaillierter in der im Abschluss befindlichen Dissertation von Martina Bross, Alternative Hamlets: Poetic Economy on Page and Stage diskutiert. 
um den Steigerungsgrad von Ironie handeln, der als Sarkasmus zu bezeichnen ist. Es ist aber auch denkbar, dass Polonius so beschränkt ist und selbst so sehr in den Dimensionen des Gebens und Nehmens befangen, dass er tender überhaupt nur im kommerziellen Sinn verstehen kann (vgl. auch die Worte „pay“ und „sterling“, die er gebraucht). Als externe Kommunikationspartner müssen wir entscheiden, ob Ironie vorliegt oder nicht; eine Entscheidung, die auf die Interpretation der Poloniusfigur und damit des ganzen Stückes Auswirkungen hat. ${ }^{16}$ Auf Seiten Shakespeares liegt also eine strategische Ambiguierung vor, die sich auf der internen Kommunikationsebene als An- oder Abwesenheit von Ironie manifestiert. Dass das Ganze auf einer lexikalischen Ambiguität, nämlich der Polysemie von tender beruht, macht den Vorgang besonders interessant.

Ich möchte noch ein weiteres Beispiel für diese erste Art der Verbindung von Ironie und Ambiguität anführen. Es geht um jene spezielle Form von Ironie, die laut Reboul auf einer doppelten Interpretation von Sätzen beruht, die einerseits als "narrative sentences“ und andererseits als „represented speech and thought“ gelesen werden können (2001: 260). Letztere nennt sie Metarepräsentationen. Ihr Beispiel aus Dickens' Great Expectations habe ich etwas erweitert. Es handelt sich um die Stelle im 18. Kapitel des ersten Buches, an der Pip, der Protagonist des Ich-Romans, von einem Rechtsanwalt mitgeteilt bekommt, dass er große finanzielle Erwartungen habe.

„I am instructed to communicate to him,“ said Mr. Jaggers, throwing his finger at me, sideways, ,that he will come into a handsome property. Further, that it is the desire of the present possessor of that property, that he be immediately removed from his present sphere of life and from this place, and be brought up as a gentleman - in a word, as a young fellow of great expectations."

My dream was out; my wild fancy was surpassed by sober reality; Miss Havisham was going to make my fortune on a grand scale.

„Now, Mr. Pip,“ pursued the lawyer, „I address the rest of what I have to say, to you. You are to understand, first, that it is the request of the person from whom I take my instructions, that you always bear the name of Pip. You will have no objection, I dare say, to your great expectations being encumbered with that easy condition. But if you have any objection, this is the time to mention it." (Dickens 2008: 125)

Der entscheidende Satz lautet: „My dream was out; my wild fancy was surpassed by sober reality; Miss Havisham was going to make my fortune on a grand scale“. Dies kann entweder als Bericht der Gedanken des erlebenden Ichs oder als Tatsa-

16 Wir haben hier also einen Sonderfall dessen, was Booth als diejenige Ironie bezeichnet, die sich durch die Annahme des Lesers ergibt, der Sprecher (Autor) könne aufgrund seiner Überzeugungen eine Aussage nicht wörtlich gemeint haben (1974: 35). Im Hamlet-Beispiel nehmen wir als Leser die Ironie des Autors an; es bleibt aber offen, ob der Sprecher (Polonius) ironisch ist. Somit ergibt sich eine Beziehung zur Thematik der unreliability, die Fludernik in ihrer Diskussion von Booth herstellt (2007: 18). Wir lesen z. B. den Brief von Mr. Collins in Jane Austens Pride and Prejudice als unfreiwillig-ironisch (bzw. als Fall von dramatic irony). Während diese Fälle aber nicht mehrdeutig sind, besteht im HamletBeispiel Ambiguität hinsichtlich der Ironie. 
chenbericht des erzählenden Ichs interpretiert werden. Erst viel später wird dem Leser unzweideutig klar, dass es sich um eine Gedanken-, d. h. Metarepräsentation gehandelt haben muss, denn Miss Havisham stellt sich nun eben nicht als die Wohltäterin des Protagonisten heraus, sondern es ist der sozial geächtete, ehemalige Sträfling Magwitch. Die Ambiguität findet sich also auf der externen Ebene der Kommunikation mit dem Leser. Wir sehen hier, dass eine Ambiguitätsauflösung, die meist auf der Basis eines einzigen Satzes oder weniger Sätze betrachtet wird, in einem Roman mehrere hundert Seiten auf sich warten lassen kann.

Die Ironie bedarf in diesem Fall der Ambiguität. Sie besteht darin, dass das genaue Gegenteil von „my wild fancy was surpassed by sober reality“ wahr ist; Pip ist in Wirklichkeit an dieser Stelle weiter ganz in seinen Wunschträumen gefangen. Wenn man Hutcheons „edge“, den Stachel oder Spott sehen will, so ist er vom Erzähler gegen sein früheres, selbstverliebtes Ich gerichtet, indem er diesen in seiner Beschränktheit exponiert. Wir haben bei dieser narrativen Ironie (als Analogon zur dramatischen Ironie, die ebenfalls auf Diskrepanz zwischen dem Wissen von Figur und Erzähler bzw. Zuschauer/Leser beruht) aber die Besonderheit, dass hier eigentlich gar niemand ironisch spricht oder sprechen muss, sondern dass die Ironie rein auf einer unironisch gebrauchten Aussage beruht, deren Gegenteil objektiv wahr ist. Es sei denn, wir würden in „my wild fancy was surpassed by sober reality“ den Ausdruck „sober reality“ als ironische Äußerung des erzählenden Ichs interpretieren. Dies ändert aber nichts daran, dass wir keine solche lokale Ironie brauchen, um die erzählerische Ironie zu konstatieren. Die Lesart von „sober reality“ als ironische Äußerung des erzählenden Ichs führt also $\mathrm{zu}$ einer doppelten Ironie.

Es erscheint lohnend, die Ambiguität der Ironie, die sich aus der Doppelung der Sprecher (der Erzähler lässt sein früheres Ich sprechen) ergibt, mit dem Begriff der Polyphonie in Verbindung zu bringen, wie er in Abwandlung von Bachtins Polyphoniemetapher von Ducrot (1984) zur Beschreibung sprachlicher Sachverhalte gebraucht wird. Ducrot unterscheidet u. a. den locuteur einer Äußerung (,derjenige, der für den Inhalt einer Äußerung verantwortlich ist“; Waltereit 2006: 63) von dem énonciateur (die „tatsächliche oder fiktive Instanz, der eine Proposition zugeschrieben wird“; 63). ${ }^{17}$ In unserem Fall besteht die Ambiguität darin, dass sowohl der Erzähler als auch der junge Pip als locuteur und énonciateur auftreten, und zwar in unterschiedlichen Kombinationen. Solange ich z. B. als Leser die narrative Ironie nicht erkenne, muss ich annehmen, dass sich der Erzähler als locuteur die (gedankliche) Äußerung „my wild fancy was surpassed by sober reality“ zu eigen macht bzw. verantwortet, deren énonciateur der junge Pip ist. Bei narrativer Ironie macht sich der Erzähler die Äußerung nicht zu eigen

17 Ducrots Instanz des sujet parlant („derjenige, der eine Äußerung materiell hervorbringt“; Waltereit 2006: 63) ist für unseren Zusammenhang von geringerer Bedeutung; angewandt auf literarische Texte ist dies in der Regel der Autor. Ich bedanke mich bei Peter Koch und Esme Winter-Froemel für den Hinweis auf Ducrots Konzept und für Literaturhinweise. 
(wir haben es also mit verschiedenen locuteurs zu tun); allenfalls verantwortet er die vorübergehende Irreführung der Leser durch die Äußerung des jungen Pip. Nehmen wir schließlich an, „sober reality“ sei die ironische Äußerung des erzählenden Ichs, so kann man annehmen, der Erzähler mache sich die Negation (bzw. das Gegenteil) dieser Äußerung zu eigen. Ironie kann als eine Form der Polyphonie angesehen werden, wobei in unserem Fall eben die Ambiguität hinzutritt, weil die Rollen von énonciateur und locuteur nicht eindeutig verteilt sind.

Auf eine spezielle Form der Ironie, die mit der doppelten Kommunikation fiktionaler Texte zusammenhängt, hat Gregory Currie hingewiesen. Currie bezeichnet ironische Äußerungen von Sprechern in fiktionalen Texten als „nested fiction“ (Currie 2006: 129), da diese Sprecher so tun, als würden sie etwas ernsthaft sagen. Laut Currie schaffen sie also eine Fiktion innerhalb der Fiktion. Wie schon erwähnt, ist zu bezweifeln, dass es sich dabei wirklich um Fiktionserzeugung in einem Sinn handelt, der mit der Hervorbringung eines fiktionalen literarischen Textes (Films, Theaterstücks usw.) vergleichbar ist. Das So-tun-als-ob der Ironie zielt darauf $a b$, dass der Hörer (Leser) die Inadäquatheit des Gesagten erkennt, während genau dies bei der Fiktionalität literarischer Texte nicht der Fall ist, wenn man von Sonderfällen wie der Parodie oder ironischen Erzählerkommentaren absieht. Anders ausgedrückt: Ironie als make-belief setzt voraus, dass die Vortäuschung als Vortäuschung erkannt wird und sich das Gemeinte vom Gesagten unterscheidet. Der ironische Sprecher spielt eine Rolle, von der ich weiß, dass er sie nur spielt, um zugleich seinen eigenen (nicht vorgetäuschten) Standpunkt zu Gehör zu bringen. Diese Differenz kann, wie ausgeführt, eine Quelle von Ambiguität sein. Bei dem Schauspieler, der eine Rolle spielt, interessiert aber normalerweise überhaupt nicht, was er als Schauspieler sagen will, zumal die Worte, die er spricht, in der Regel nicht seine eigenen sind. Die Illusionsstörung ist im Theater der Sonderfall, bei der Ironie Bedingung. Im Theater stellt sie eine Gefährdung des für die Fiktion notwendigen Fürwahrhaltens (willing suspension of disbelief) dar, bei ironischem Sprechen darf dagegen die vorgetäuschte Rolle gar nicht für wahr gehalten werden; Fiktion ist in diesem Sinn nicht wirklich gegeben.

Nun kann Ironie auch ins Spiel kommen, wenn der fiktionale Sprecher seine Äußerung nicht ironisch verstanden wissen will, also keine Rolle vortäuscht. Wir haben dies schon bei dem Beispiel aus Great Expectations gesehen, wo sich die Ironie daraus ergibt, dass das Gegenteil der unironischen Aussage des erlebenden Ichs in der Welt der Fiktion objektiv wahr ist. Currie erwähnt solche Fälle nicht, sondern hat einen anderen Sonderfall der Ironie auf der Basis von Äußerungen fiktionaler Figuren im Blick, der für das Verhältnis von Ironie und Ambiguität von Belang ist. Er führt aus, dass in Bühnen- und Filmdialogen immer wieder Äußerungen fallen, die von ihrem jeweiligen fiktionalen Sprecher nicht ironisch gemeint sind, die aber, hörte man sie im wirklichen Leben, nur als ironische Äußerungen verstanden werden könnten. Currie nennt dieses Phänomen „suppressed irony“ (Currie 2006: 111, 129), was ich nicht für ganz glücklich halte, denn 
hier wird keine Ironie unterdrückt. Er führt als Beispiel einen Dialog von Nydia Westman und Bob Hope in der Horrorfilmkomödie The Cat and the Canary $(1940)^{18}$ an: „,Don't these big empty houses scare you?" ,Not me, I was in vaudeville““ (129). Currie erklärt, dass solche fiktionalen Sprecher ganz ernsthaft „absurdly defective perspectives“ einnehmen, „and that is part of what makes their remarks funny" (130). ${ }^{19}$ Während diese Beschreibung auch für viele Fälle erzählerischer und dramatischer Ironie zutrifft (Pips Sicht in Great Expectations ist in der Tat „absurdly defective“), besteht der entscheidende Unterschied allerdings darin, dass die Ironie im „big empty houses“-Beispiel nicht innerhalb der Fiktion etabliert wird, sondern außerhalb. Wenn sie denn etabliert wird, denn das ist keineswegs ausgemacht, wie Currie in seinem bereits paraphrasierten Statement erkennen lässt: „the actor just said something which, if uttered in real life, could only be uttered ironically" (130).

Man könnte hier also eher von „hypothetical irony“ sprechen, zumal nicht ganz klar ist, worin die Ironie besteht. Ambiguität ist insofern im Spiel, als eine fiktionale Äußerung, die nicht ironisch ist, in einem nichtfiktionalen Zusammenhang ironisch wäre oder sein könnte. Wenn wir die Antwort „Not me, I was in vaudeville" als ironisch auffassen, so ist es die Ironie des Autors, ${ }^{20}$ der diesen Dialog fingiert hat, nicht die Ironie des Sprechers im Dialog. Er macht sich über die (offenbar nicht mehr populären) Vaudeville-Aufführungen lustig. Dies wäre (auch wenn Currie darauf nicht hinweist) ein Beispiel für die doppelte Kommunikation, wie sie fiktionalen Texten eignet. Während aber in dem Great Expectations-Beispiel innerhalb der Fiktion ein Rahmen dafür geschaffen wird, eine Äußerung als erzählerische Ironie zu erkennen (analog zur dramatischen Ironie), bleibt dies im vorliegenden Fall aus. Es würde sich also um einen der oben genannten Sonderfälle literarischer Fiktion handeln: Die sprechenden Figuren sind Fiktionen des Autors, die nicht als bloß vorgetäuscht zu durchschauen sind, wenn wir folgen wollen. Gleichzeitig kann der Autor ihnen Sätze in den Mund legen, die von ihm (nicht von der Figur) ironisch gemeint sind; er spielt also für einen Moment seine eigene Figur und stört damit in gewisser Hinsicht die Fiktion. Ironie erzeugt hier eine besondere Form der Ambiguität, die darin besteht, dass ich eine Äußerung als fiktional (von der Figur gesprochen) und nichtfiktional (vom Autor gesprochen) erkenne. Wo man fiktionale Vermittlungsinstanzen hat (Erzähler usw.), kann dies noch vielschichtiger werden.

18 Curries Jahresangabe; laut Internet Movie Database stammt der Film aus dem Jahr 1939, vgl. <http://www.imdb.com/title/tt0031143/?ref_=nv_sr_1>; vgl. auch <http://www.the guardian.com/film/pictures/image/0,8545,-10104248057,00.html $>$.

19 Er erklärt das „big empty houses“-Beispiel damit, dass Sprecher wie der zitierte „[are] seriously asserting that their vaudeville experience has prepared them for grim and ghostly buildings" (Currie 2006: 130). Das halte ich nicht für plausibel. Der Witz ergibt sich m. E. nur dann, wenn man den Sprecher so versteht, dass die Vaudeville-Bühne ihn an leere Häuser gewöhnt habe.

20 Gegebenenfalls des Erzählers in narrativ vermittelten Dialogen. 
Anders als Currie meint, ist diese Art von Dialog nicht auf „staged or filmed humour" (129) beschränkt. Das obige Beispiel von P. G. Wodehouse (der allerdings auch viel Erfahrung mit komischen Bühnendialogen besaß) zeigt dies. Wenn Lord Ickenham auf die Frage "Jamaica?" antwortet „No, she went of her own free will“, so scheint es uns, als habe er, aufgrund seines Verhörers, eine absurd falsche Perspektive eingenommen. In diesem Fall bewerten wir die Äußerung nicht als ironisch. Dies wird sie erst, wenn wir annehmen, er habe seinen Gesprächspartner bewusst missverstanden und spiele den Verhörer nur. Der Leser, der die Äußerung nicht verhört, sondern sich den Verhörer überhaupt erst erschließen muss, nimmt die Ironie wahr; ob diese aber auch innerhalb der Fiktion vorliegt, bleibt offen. Wir haben es also einmal mehr mit einer mehrfachen Ambiguität zu tun.

Eine besondere Verbindung von Ironie und Ambiguität findet sich schließlich in Fällen, in denen ein (fiktionaler) Hörer auf eine ironische Äußerung mit einer mehrdeutigen Äußerung reagiert bzw. die ironische Äußerung als mehrdeutig auffasst. Hier geht der Hörer zwar nicht direkt auf die Ironie ein, aber er zeigt, dass er sie erkannt hat, weil er sie als Spiel auffasst, in das er einsteigt. Die beiden gewitzten Diener-Narren Speed und Lance in Shakespeares The Two Gentlemen of Verona liefern ein Beispiel dafür. ${ }^{21}$ Speed redet seinen Kollegen übertrieben ehrfürchtig mit „Signor Lance“ an; er tut also so, als sei Lance ein Herr: „What news with your mastership?" Lance tut seinerseits so, als habe er den freundlichen Spott nicht verstanden, weil er „mastership“ eine andere Bedeutung gibt: „With my master's ship? Why, it is at sea“. Dass der Verhörer hier nur gespielt ist, zeigt sich in der Reaktion Speeds, der seinen Gesprächspartner gut kennt: „Well, your old vice still: mistake the word" (3.1.275-77). Speed erkennt Lance also, wie sich ein anderer Narr bei Shakespeare selbst charakterisiert, als "corrupter of words“. ${ }^{22}$ Den Spott kann Lance parieren, indem er geschickt mittels eines als Verhörer getarnten Wortspiels das Thema wechselt. Speed pariert dann im Gegenzug mit einem Wortspiel, indem er einerseits auf Lances altes „Laster“, das WortMissverstehen, Bezug nimmt (und das Missverstehen damit als bewusste Boshaftigkeit, nicht als Dummheit erkennt) und andererseits Lance als „old vice“ bezeichnet und damit als gewitzt-komische Verkörperung des Lasters aus dem Moralitätenspiel. ${ }^{23}$ Eine vielleicht ungeplante Ambiguität in der doppelten Kommu-

21 Ich bin Angelika Zirker für den Hinweis auf diese Stelle ebenso dankbar wie für weitere Anregungen und kritisches Feedback.

22 Feste bezeichnet sich so gegenüber Viola in Treelfth Night (3.1.35).

23 Die Verbindung von Vice und Wortspiel lässt Richard III bei Shakespeare erkennen, der sich mit dem Vice identifiziert: „Thus like the formal Vice, Iniquity, / I moralize two meanings in one word" (3.1.82-83). William C. Carroll geht in der Arden-Ausgabe von The Two Gentlemen of Verona nicht darauf ein, dass Speed hier zunächst Lances altes Laster meint (und nur sekundär auf die Figur aus dem Moralitätenspiel anspielt). Auch ist ganz unverständlich, dass Carroll meint, „his verbal mistakings, unlike those of the Vice, are usually inadvertent rather than self-conscious" (2004: 221; Anmerkung zu 3.1.278). 
nikation des Dramas zeigt sich darin, dass zwar Speed und Lance sehr genau wissen, was sie tun, aber der Zuschauer oder Leser der Szene womöglich nicht merkt, was gespielt wird. So ist im Oxford Handbook of Shakespeare's Poetry zu lesen: „Lance fails to grasp the irony of Speed's address“ (Ferguson 2013: 80), was umso mehr erstaunt, als Lance sich wenig später nicht nur als Meister homophoner Wortspiele, sondern auch des virtuosen Umgangs mit Synonymen zeigt, wenn er „nameless virtues“ als „bastard virtues“ bezeichnet (3.1.309-10).

\section{Ambiguität aufgrund der zusätzlichen kommunikativen Bedeutung einer ironischen Äußerung}

Meine weiteren vorgeschlagenen Arten der Beziehung von Ambiguität und Ironie kann ich sehr viel knapper behandeln. Die zweite beruht darauf, dass Ironie, die ja neben einer bestimmten Sprachverwendung auch durch einen Effekt gekennzeichnet ist (Hutcheons edge), eine Äußerung mit Bedeutung anreichert. Es ist also nicht nur so, dass Ironie häufig mit Ambiguität arbeitet oder einhergeht; der ironische Sprachgebrauch selbst verändert die Bedeutung zumindest in pragmatischer (wenn nicht strikt in semantischer) Hinsicht. Anders ausgedrückt: Es macht einen Unterschied, ob ich sage „my wild fancy was surpassed by an even wilder one“ oder "my wild fancy was surpassed by sober reality“, auch wenn, wie wir soeben angenommen haben, Letzteres semantisch die Bedeutung von Ersterem hat. Das, was bei der Ironie hinzutreten kann, ist also der durch das Aufrufen (und Negieren) des Literalsinns bzw. der durch das im Negieren erfolgende Aufrufen des Literalsinns ausgelöste Effekt. Dieser kann in einer Implikatur bestehen: ${ }^{24} \mathrm{Im}$ vorliegenden Fall ist etwa die pragmatische Bedeutung der Äußerung darin zu sehen, dass der junge Pip als immer noch realitätsblind und selbstbezogen charakterisiert wird.

Ich halte es für gerechtfertigt, hier von einer Form der Ambiguität zu sprechen, weil durch dieselbe Äußerung mehrere Interpretationen ausgelöst werden. Ambiguität ergibt sich also nicht nur dadurch, dass eine Äußerung ironisch sein kann oder nicht (bzw. es für den einen Hörer ist und für den anderen nicht). Eine als ironisch erkannte oder interpretierte Äußerung ist darüber hinaus auch dadurch mehrdeutig, dass zum einen die wörtliche Bedeutung ihres Gegenteils aufgerufen wird und zum anderen eine zusätzliche Bedeutung, die der Auslöser des für die Ironie nötigen Effekts (oder edge) ist, also klassischer Weise die (strenge oder milde) Verspottung des Gegenübers (als Korrektiv) und auch die Selbstdistanzierung oder -demütigung. Im vorliegenden Beispiel aus Great Expectations wird durch die Wortwahl „sober reality“, die ironisch negiert wird, einerseits vermittelt, dass Pip immer noch Illusionen nachjagt, andererseits wird der Kon-

24 Zur Beschreibung der Ironie im Kontext des Griceschen Modells siehe Dynel (2013). Ich bezweifle allerdings Dynels verabsolutierende Annahme, ,irony [...] generates conversational implicature invariably carrying negative evaluation“ (403). 
trast aufgerufen, der Pips Irrtum umso deutlicher hervortreten lässt und damit den Spott des erzählenden Ichs sichtbar macht. (Die nüchterne Realität der Desillusionierung wird für Pip kommen; die ironische Stelle hier kann also auch als Vorausdeutung gelesen werden.) Der Kontrasteffekt wird durch die Lesart als Gedankenbericht verstärkt. Ich halte es für gerechtfertigt, hier von Ambiguität (im Sinne einer Mehrfachbedeutung) zu sprechen, denn ich kann mich als Leser immer entscheiden, ob die ironische Aussage vornehmlich die Bedeutung hat, das Gegenteil auszudrücken, oder ob sie vornehmlich die pragmatische Bedeutung der Distanzierung, Verspottung usw. hat.

$\mathrm{Zu}$ den z. T. sprechakttheoretisch zu beschreibenden Effekten zählt auch, dass der Sprecher selbst durch die Ironie charakterisiert wird. Ein Beispiel hierfür findet sich in dem geistlichen Sonett „At the round earth's imagined corner" von John Donne. Darin heißt es in den letzten Zeilen: „here on this lowly ground / Teach me how to repent, for that's as good / As if thou'dst sealed my pardon with thy blood" (Donne 2010: 536, Z. 12-14).

Die Ironie besteht hier darin, dass der Sprecher, der sich an Gott bzw. Christus wendet, nicht zu wissen scheint, dass seine Begnadigung längst durch das Blut des Angesprochenen besiegelt ist. In diesem Fall würde es sich um eine Form dramatischer Ironie handeln (unser Fall 1.2): der Leser denkt daran, dass im christlichen Kontext der Kreuzestod Erlösung bedeutet, während der Sprecher dies nicht zu wissen scheint. Eben diese Charakterisierung des Sprechers als offenbar unwissend ist aber ein Bestandteil von Donnes Aussage im Gedicht: Der Sprecher muss, damit er die Reue lernt, gewissermaßen vergessen, dass er bereits begnadigt ist. Eine alternative Lesart besteht darin, dass der Sprecher selbst ironisch ist, denn er weiß von dieser Erlösungshandlung. In diesem Fall hätte die Ironie den Effekt, ihn nicht ohne Schlauheit im Gespräch mit Gott zu zeigen.

\section{Ambiguität aufgrund des Simulationscharakters einer ironischen Äußerung}

Meine dritte Kategorie in der Beziehung von Ambiguität und Ironie beruht darauf, dass Ironie, seit der klassischen Antike (aber bis in Theorien der Gegenwart), ${ }^{25}$ als eine Form der Vortäuschung angesehen worden ist, und zwar entweder als dissimulatio oder als simulatio, also in der wertmäßigen Herabstufung der eigenen Position, z. B. durch understatement (litotes) (Müller 1989: 190-91), oder in der „positiven Vortäuschung einer eigenen, mit einer Meinung der Gegenpartei übereinstimmenden Meinung“ (Lausberg 1973: Par. 902.2 nach Quintilian

25 Vgl. Lapp (1992). Zur Diskussion, ob es sich bei Ironie um pretence handelt oder um echoic mention (einer Äußerung, von der sich der ironische Sprecher distanziert), siehe z. B. Curry (2006) einerseits und z. B. Sperber/Wilson (1981) und Wilson (2006) andererseits. Für das Verhältnis von Ironie und Ambiguität sind diese Definitionsunterschiede i. d. R. nicht entscheidend. Sie beruhen letztlich darauf, welche Funktion ironischer Äußerungen betont wird; in keinem Fall sind pretence und echoic mention hinreichende Definitionen. 
6.3.85). Ein Beispiel für dissimulatio ist Mark Antonys scheinbar selbstkritische Äußerung „I am no orator“ in seiner berühmten Rede im dritten Akt von Julius Caesar (3.2.219; dazu Knape/Winkler [im Druck]). Ein Beispiel für die zweite Variante ist seine Aussage „For Brutus is an honorable man“ (3.2.84 u. ö.), die vortäuschen soll, dass er der Meinung des Volkes ist. Beide Varianten der Vortäuschung können entweder dazu dienen, den eigenen Standpunkt zu verbergen (d. h. der andere soll nicht merken, dass Ironie vorliegt) oder, und dies ist die eigentliche rhetorische Ironie, es soll vielmehr durch dis/simulatio die Gegenposition als falsch hervortreten.

Hier bemerken wir schon, dass die Vortäuschung mit der Sichtbarmachung der Vortäuschung einhergeht. ${ }^{26}$ Dabei sehen wir von dem sich der Lüge nähernden Fall der Verbergung der Ironie ab: Antonys Aussage ist keine Lüge, weil die eigene Partei bzw. der eingeweihte Leser merkt, dass Ironie vorliegt. Auch in diesem Fall paaren sich Vortäuschung und Sichtbarmachung der Vortäuschung, nur dass sie hier auf verschiedene Kommunikationspartner verteilt sind, was zurückverweist auf unsere erste Verbindung von Ironie und Ambiguität. Bekanntlich gibt es Hinweise (sogenannte Ironiesignale), welche die Täuschung als solche erkennen lassen und damit aufheben. Dies kann durch Intonationsmuster (etwa Übertreibungen) geschehen, welche auf die ironische dis/simulatio hinweisen, oder durch die Wiederholung der Äußerung, die in der Hamlet-Passage besonders raffiniert verwendet wird, weil sie einerseits suggeriert, dass Polonius ironisch spricht, andererseits aber auch dazu dient, ihn stereotypisch als geschwätzigen Alten darzustellen. Die Wiederholung ist auch Ironiesignal in der erwähnten Rede Mark Antonys in Julius Caesar (vgl. Knape/Winkler [im Druck] 64).

Die Verbindung zur Ambiguität ergibt sich nun dadurch, dass die Vortäuschung als dis/simulatio zusammengeht mit der Wahrheit. Letztere ist nicht identisch mit dem Ironiesignal, aber das Signal weist auf sie hin, weil es zeigt, dass Ironie keine Lüge bedeutet. Bei der Ironie wird, wie bei der Lüge, nicht das Gesagte gemeint. Aber im Gegensatz zur Lüge besteht keine Täuschungsabsicht. Wir haben es hier mit einer anderen Art von Ambiguität zu tun als in den bisher besprochenen Fällen, nämlich mit dem Miteinander von sich eigentlich ausschließenden Bedeutungen, nun nicht auf semantischer Ebene, sondern, wie ich vorschlagen möchte, auf der Ebene von Fiktonalität versus Nichtfiktionalität. Lapp (1992) bezeichnet Ironie als simulierte Sprechhandlung; ich würde es so formulieren, dass durch die Simulation eines Literalsinns etwas der Fiktion zumindest Analoges geschaffen wird, das gleichzeitig nichtfiktional unterlaufen wird. Unser Ausgangsbeispiel Defoe kann hierfür erhellend sein: Der „True-Born Englishman" ist, wenn er als Wirklichkeitsbeschreibung aufgefasst wird, eine Fik-

26 In Gricescher Terminologie findet sich dies bei Dynel so ausgedrückt: „[I]rony is intentionally produced by the speaker in the form of overt untruthfulness, based on flouting the first maxim of Quality, to generate conversational implicature, which is to be duly recognized by the hearer" (2013: 404). 
tion; er existiert nur in der möglichen, aber nicht wirklichen (und von Defoe auch nicht gewünschten) Welt eines reinrassigen England; nichtfiktional und ironisch gesprochen beschreibt der Ausdruck etwas wirklich Anzutreffendes, nämlich den verblendeten Stolz auf die eigene Rasse.

Wir können feststellen, dass uns die Beschreibung des Verhältnisses von Ironie und Ambiguität hilft, die Beziehung von Faktualität und Fiktionalität zu reflektieren. Ironie ist ein Grenzphänomen, das zugleich innerhalb und außerhalb der durch die Fiktion beschriebenen möglichen Welt existiert. Wir sehen dies bei dramatischer und narrativer Ironie, die durch das Miteinander von interner und externer Kommunikation entsteht. Auch auf Seiten des ironischen Sprechers findet sich diese Doppelung: Er spielt eine Rolle, schafft zumindest für einen Moment eine Fiktion, bezieht sich aber doch, anders als die Fiktionen von Literatur, Theater und Film, notwendigerweise und unmittelbar auf die wirkliche Welt. Ich möchte schließen mit dem Verweis auf eine ironische Metareflexion über das Verhältnis von Fiktion und Wirklichkeit aus dem Munde des Spötters Touchstone in Shakespeares As You Like It:

TOUCHSTONE When a man's verses cannot be understood, nor a man's good wit seconded with the forward child, understanding, it strikes a man more dead than a great reckoning in a little room. Truly, I would the gods had made thee poetical.

AUDREY I do not know what poetical is. Is it honest in deed and word? Is it a true thing?

TOUCHSTONE No, truly; for the truest poetry is the most faining [feigning], and lovers are given to poetry, and what they swear in poetry may be said, as lovers, they do feign. $(3.3 .10-19)$

Bei Touchstone verbinden sich Ironie und Paradox, wenn er sagt „The truest poetry is the most feigning" (ironisch deshalb, weil auch das Gegenteil wahr ist; vgl. Leimberg 1979). Sein Satz ist mehrdeutig, weil er einerseits heißen kann, dass noch die ,wahrste' Dichtung letztlich eine Täuschung (Fiktion) ist, andererseits aber auch die am meisten vortäuschende, fingierte Dichtung die,wahrste ist. So verweist uns die Ironie auf eine Ambiguität, die darin besteht, dass Fiktion als Fiktion die Wahrheit sagt.

\section{Literatur}

Bauer, Matthias, und Sigrid Beck (2014) „On the Meaning of Fictional Texts“. Approaches to Meaning: Composition, Values, and Interpretation. Hgg. Daniel Gutzmann, Jan Köpping und Cécile Meier. Leiden: Brill. 250-75.

Bauer, Matthias, Joachim Knape, Peter Koch und Susanne Winkler (2010) „Dimensionen der Ambiguität“. Zeitschrift für Literaturwissenschaft und Linguistik (LiLi) 40.158: 7-75.

Behler, Ernst (1998) „Ironie“. Historisches Wörterbuch der Rhetorik. Hg. Gert Ueding. Band 4. Tübingen: Niemeyer. 599-624. 
Booth, Wayne C. (1974) A Rhetoric of Irony. Chicago: The Univ. of Chicago Press. Currie, Gregory (1990) The Nature of Fiction. Cambridge: Cambridge Univ. Press.

- (2006) „Why Irony is Pretence“. The Architecture of the Imagination: Nerw Essays on Pretence, Possibility, and Fiction. Hg. Shaun Nichols. Oxford: Oxford Univ. Press. 111-33.

Davies, David (2007) Aesthetics and Literature. London: Continuum.

Defoe, Daniel (2003) „The True-Born Englishman: A Satyr [1700]“. Satire, Fantasy and Writings on the Supernatural by Daniel Defoe. Hgg. W. R. Owens und P. N. Furbank. Bd. 1. London: Pickering and Chatto. 77-122.

Dickens, Charles (2008) Great Expectations [1861]. Hgg. Margaret Cardwell und Robert Douglas-Fairhurst. Oxford World's Classics. Oxford: Oxford Univ. Press.

Donne, John (2010) The Complete Poems. Hg. Robin Robbins. Harlow: Longman.

Ducrot, Oswald (1984) „Esquisse d'une théorie polyphonique de l'énonciation“. Le dire et le dit. Paris: Editions de Minuit. 171-233.

Dynel, Marta (2013) „Irony from a Neo-Gricen Perspective: On Untruthfulness and Evaluative Implicature“. Intercultural Pragmatics 10.3: 403-31.

Ferguson, Margaret (2013) „Fatal Cleopatras and Golden Apples: Economies of Wordplay in Some Shakespearean ,Numbers"“. The Oxford Handbook of Shakespeare's Poetry. Hg. Jonathan F. S. Post. Oxford: Oxford Univ. Press. 77-94.

Fludernik, Monika (2007) „Interfaces of Language: The Case of Irony“. Irony Revisited: Spurensuche in der englischsprachigen Literatur. Hgg. Thomas Honegger, Eva-Maria Orth und Sandra Schwabe. Würzburg: Königshausen und Neumann. 11-26.

Hutcheon, Linda (1994) Irony's Edge: The Theory and Politics of Irony. London: Routledge.

Knape, Joachim, und Susanne Winkler (im Druck) „Strategisches Ambiguieren, Verstehenswechsel und rhetorische Textleistung: Am Beispiel von Shakespeares Antony-Rede". Ambiguität: Produktion und Rezeption / Ambiguity: Production and Perception. Hg. Susanne Winkler.

Lapp, Edgar (1992) Linguistik der Ironie. Tübingen: Narr.

Lausberg, Heinrich (1973) Handbuch der literarischen Rhetorik [1960]. 2. Aufl. München: Hueber.

Leimberg, Inge (1979) „The Truest Poetry Is the Most Feigning: Dichtung und Wahrheit in As You Like It". Studien zur englischen Philologie: Edgar Mertner zum 70. Geburtstag. Hgg. Herbert Mainisch und Dieter Rolle. Frankfurt: Lang. 51-81.

Müller, Wolfgang G. (1989) „Ironie, Lüge, Simulation und Verwandtes“. Zur Terminologie der Literaturwissenschaft. Hg. Christian Wagenknecht. Stuttgart: Metzler. 189-208.

Nugent, Elliott (1939) Dir. The Cat and the Canary. Original play by John Willard [1922]. Los Angeles: Paramount Pictures.

Oxford English Dictionary Online (OED). Oxford: Oxford Univ. Press. (http:// www.oed.com/) 
Quintilianus, Marcus Fabius Quintilianus (1988) Ausbildung des Redners: Zwölf Bücher. 2. Aufl. Hg. und übers. Helmut Rahn. Darmstadt: Wissenschaftliche Buchgesellschaft.

Reboul, Anne (2001) „Represented Speech and Thought and Auctorial Irony: Ambiguity and Metarepresentation in Literature". Quitte ou Double Sens: Articles sur l'ambiguitté offerts à Ronald Landheer. Hgg. Paul Bogaards, Johan Rooryck und Paul J. Smith. Amsterdam: Rodopi. 253-77.

Shakespeare, William (2006) As You Like It. Hg. Juliet Dusinberre. London: Thomson Learning.

- (1982) Hamlet. Hg. Harold Jenkins. The Arden Shakespeare. London: Methuen.

- (2006) Julius Caesar. Hg. David Daniell. The Arden Shakespeare. London: Thomson Learning.

- (2009) King Richard III. Hg. James R. Siemon. The Arden Shakepsare. London: Bloomsbury Publishing.

- (2008) Treelfth Night. Hg. Keir Elam. The Arden Shakespeare. London: Cengage Learning.

-(2004) The Trwo Gentlemen of Verona. Hg. William C. Carroll. The Arden Shakespeare. London: Bloomsbury Publishing.

Sperber, Dan, und Deirdre Wilson (1981) „Irony and the Use-Mention Distinction“. Radical Pragmatics. Hg. Peter Cole. New York: Academic Press. 295-318.

- (1995) Relevance: Communication and Cognition. 2. Aufl. Malden: Blackwell.

Waltereit, Richard (2006) Abtönung: Zur Pragmatik und historischen Semantik von Modalpartikeln und ibren funktionalen Äquivalenten in romanischen Sprachen. Tübingen: Niemeyer.

Wilson, Deirdre (2006) „The Pragmatics of Verbal Irony: Echo or Pretense?“ Lingua 116: 1722-43.

Winter-Froemel, Esme, und Angelika Zirker (2010) „Ambiguität in der SprecherHörer-Interaktion: Linguistische und literaturwissenschaftliche Perspektiven“. Zeitschrift für Literaturwissenschaft und Linguistik 40.158: 76-97.

- (im Druck) „Ambiguity in Speaker-Hearer-Interaction: A Parameter-Based Model of Analysis“. Ambiguität: Produktion und Rezeption/Ambiguity: Production and Perception. Hg. Susanne Winkler.

Wodehouse, P. G. (1966) Uncle Dynamite [1948]. Harmondsworth: Penguin. 